Paper

\title{
Semantic Concept Detection based on Spatial Pyramid Matching and Semi-supervised Learning
}

\begin{abstract}
Yoshihiko Kawai (member) $^{\dagger}$, Mahito Fujii (member) ${ }^{\dagger}$
Abstract Analyzing video for semantic content is very important for finding the desired video among a huge amount of accumulated video data. One conventional method for detecting objects depicted in video is called the bag-of-visual-words method, and is based on local feature occurrence frequencies. We propose a method that improves on the detection accuracy of traditional method by dividing video frames into overlapped sub-regions of various sizes. The method computes local and global features for each of these sub-regions to reflect spatial positioning in the feature vectors. These changes ensure that the method is resistant to variations in the size and position of objects appearing in the video. We also propose a training framework based on semi-supervised learning that uses a small number of labeled data points as a starting point and generates additional labeled training data efficiently, with few errors. Experiments using a video data set confirmed improved detection accuracy over earlier methods.
\end{abstract}

Key words: Video semantic content, bag-of-visual-words, spatial pyramid matching, random forests, semi-supervised learning

\section{Introduction}

With the increasing speed of networks and the spread of digital video recording devices in recent years, it has become common for individuals to hold large amounts of video data. This situation has created a demand for technology enabling users to search for desired video quickly. To search through large amounts of video data effectively requires analysis of the semantic content of the video, rather than just physical characteristics such as color or texture. One method for analyzing video content is general object recognition, which detects objects and events (referred to as semantic concepts) that appear in the video. General object recognition uses machine learning to determine whether specific concepts are present in the video, with training data that has been labeled to indicate the names of concepts. By changing the training data, it can be adjusted to handle a variety of concepts.

The most common approach used for general object recognition is called bag-of-visual-words $(\mathrm{BoVW})^{1) 2)}$. The BoVW method extracts keyframes from the video, and calculates feature vectors based on the frequency of local features such as $\mathrm{SIFT}^{3)}$ and $\mathrm{SURF}^{4)}$. These are then classified using a method such as support vector machines to determine whether the specific concept ap-

Received November 22, 2012; Revised January 28, 2013; Accepted February 22, 2013

$\dagger$ Science and Technical Research Laboratories, NHK (1-10-11 Kinuta, Setagaya-ku, Tokyo, 157-8510, Japan) pears in the keyframe. The effectiveness of the BoVW framework is widely recognized and it is used in a variety of techniques such as image classification, image search and image analysis ${ }^{5) 6) 7}$.

BoVW is an extremely effective approach, but several issues still remain. One issue is that the spatial position of concepts appearing in the video frame is not reflected in the feature vectors. This is because a histogram of local feature frequencies for the entire image is calculated. To address this, a method called spatial pyramid matching (SPM) has been proposed ${ }^{8) 9}$. SPM partitions images into several sub-divisions and computes feature vectors for each sub-divisions. An earlier technique proposed dividing images into $2 \times 2$ or $1 \times 3$ sub-regions, based on experience and preliminary experiments ${ }^{10)}$.

Another issue is how the training data is created. Normally, an annotator will view the video, decide whether the desired semantic concepts are present or not, and apply labels. Labels for a positive example are applied if a particular concept is present, and for a negative example if not present. To train highly accurate classifiers, a wide variation of positive example data is needed, and for ordinary video analysis, several hundred to several thousand hours of training video data is prepared. For example, to produce an accurate classifier for automobiles, the training data should include positive examples of various models, orientations, and with various positions and sizes. Labels must also be applied for each semantic concept handled, so if there are many concept categories, this work can become ex- 
tremely expensive. To reduce the cost of this labeling work, labeled data sets for research purposes have been published ${ }^{112)}$, but if no data set exists for the desired concept, this labeling must still be done manually. At TRECVID ${ }^{13)}$, which is an evaluation workshop on video retrieval, a Web application is being used to perform collaborative annotation ${ }^{14)}$ among several research organizations. The training data is created more efficiently by having many people work on it, but there are still issues such as ambiguities in making decisions for the annotators (e.g. for concepts such as "Boy" or "Teenager"), and errors made when applying labels. Another approach to reduce the labeling cost is to use semi-supervised learning. Semi-supervised learning enables to build a classifier by using a small amount of labeled data with a large amount of unlabeled data ${ }^{15)}$. One of the major algorithms is self-training ${ }^{16) 17)}$. In self-training, a classifier is trained with the labeled data and then the unlabeled data is classified by the trained classifier. The unlabeled data with predicted labels are added to the training data set and the classifier is retrained. The procedure is repeated until a termination condition is satisfied.

In this paper, we propose a method for detecting semantic concepts based on the BoVW framework. To solve the drawbacks of traditional method, we propose two novel ideas. The first is an extended SPM to calculate the feature vector which is resistant to variations in the size and position of concepts. The second is a training framework based on semi-supervised learning, which uses a small set of labeled data items as a starting point and creates training data efficiently, with few errors.

The proposed method calculates feature vectors based on global features, taking into account larger regions, in addition to the local features used in earlier methods. Feature vectors are computed for keyframes selected from each shot in the video data. These keyframes are then partitioned based on the similar idea as used with SPM, and local and global features of each region are combined to compute the feature vector for the whole image. This incorporates spatial information for the keyframe image into the feature vector. Rather than simply partitioning with various shapes and sizes, partitions are arranged with some overlap so that the feature vector is robust against variations in the size or position of concepts. The method uses the random forests method ${ }^{18)}$ for machine learning, which is fast for both training and prediction processing. The new framework based on semi-supervised learning is used for generating training data. The annotator is only required to label a part of the data, which is presented to him/her by the system. Our framework can greatly reduce the amount of time required and the incidence of errors. Reducing the amount of work also makes it possible for a single person to perform the annotating, so the standards for determining the labeling can be more consistent. In experiments, we evaluated detection accuracy using the approximately 800 hours of the TRECVID data set ${ }^{13}$ to verify the effectiveness of the proposed method. We also verified the effectiveness of the proposed training framework in experiments using approximately 10,000 hours of television programs.

\section{Proposed Method}

An overview of the proposed method is shown in Fig. 1. First, the input video is divided into shots, and then a keyframe is extracted from the beginning of each shot and used to represent the shot. The method described in Kawai et al. ${ }^{19)}$ is used for detecting shot boundaries. Here, the earlier studies reported that the detection accuracy was improved by analyzing more than one keyframe for each shot ${ }^{5)}$. Our method also can be extended to use multiple keyframes. Next, local and global features are calculated from the keyframes. Local features are obtained by using the BoVW approach based on two algorithms: $\mathrm{SIFT}^{3)}$ and $\mathrm{SURF}^{4)}$. Global features are calculated using color moments, the Haar wavelet, and local binary patterns $(\mathrm{LBPs})^{20)}$. These local and global features are aggregated over block regions of various sizes and then combined to form a feature vector for the entire frame. Finally, the feature vectors are classified by a random forest classifier to determine whether the target concept appears in the shot. Actually, the classifier returns likelihood score for each input keyframe. The random forest classifier is assumed to have been trained in advance using training data labeled with concept names. The details of each process are described below.

\subsection{Local Features}

The proposed method calculates local features based on the combination of $\mathrm{SIFT}^{3)}$ and $\mathrm{SURF}^{4)}$ algorithms. The effectiveness of combining different kinds of local features has been verified by various existing stud$\operatorname{ies}^{2122)}$. We also combine two algorithms to capture visual characteristics of keyframes more accurately. The codebook for vector quantization is generated using the $k$-means method to cluster feature descriptors extracted 


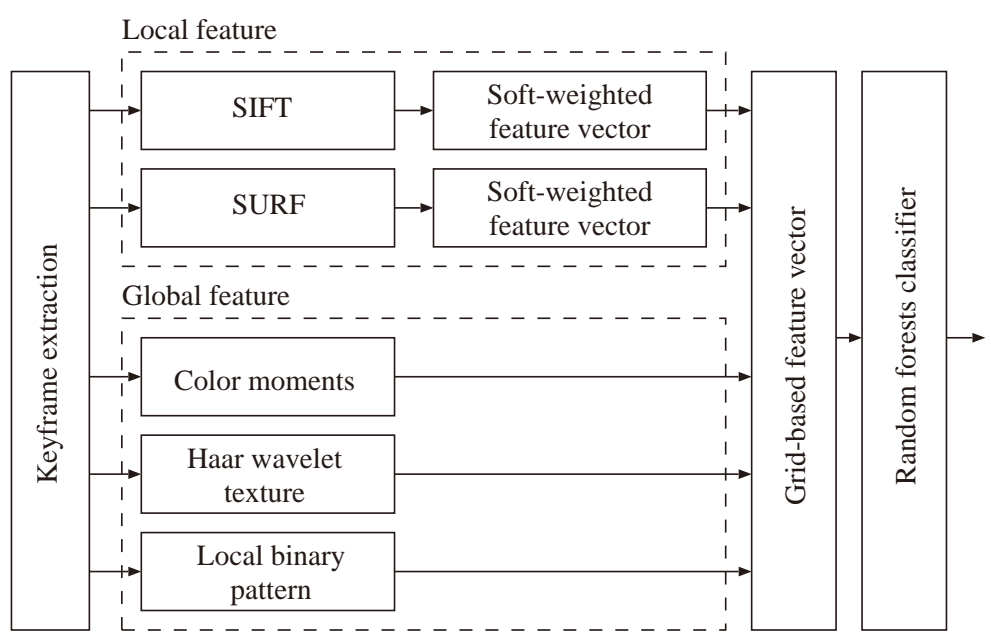

Fig. 1 Overview of semantic indexing method.

from the training data. The representative vector of each cluster is selected as a codeword for the codebook. Separate codebook is prepared for SIFT and SURF. The local feature vectors are calculated using a weighting method based on the distances between codewords and feature descriptors ${ }^{23)}$. This differs from the conventional approach, where a single descriptor is allocated to a single codeword, and instead allows a single descriptor to be associated with multiple codewords. If $D$ is the total number of codewords in the codebook, it calculates a $D$-dimensional local feature vector $\left(t_{1}, \ldots, t_{k}, \ldots, t_{D}\right)$. The vector elements $t_{k}$ are calculated with the formula

$$
t_{k}=\sum_{i=1}^{S} \sum_{j=1}^{Q_{i}} \frac{1}{2^{i-1}} \operatorname{sim}\left(p_{j}, w_{k}\right),
$$

where $Q_{i}$ is the total number of feature descriptors having $w_{k}$ as a codeword whose distance is close to the $i$-th item and $\operatorname{sim}\left(p_{j}, w_{k}\right)$ is the degree of similarity between feature descriptor $p_{j}$ and codeword $w_{k}$. S is a constant expressing how many of the closest codewords should be considered. We set $S=4$, as in as in Jiang et al. ${ }^{23)}$.

\subsection{Global Features}

The proposed method uses three types of global feature. These features were selected considering both the preliminary experimental result and the calculation cost.

\section{(1) Color Moments}

The proposed method transforms the input image into the HSV and Lab color spaces and then calculates the average pixel value $\mu_{c}$, the standard deviation $\sigma_{c}$, and the cube root of skewness $s_{c}$ for each component $c$ $(c \in\{h, s, v, l, a, b\})$. The formulae used are as follows:

$$
\mu_{c}=\frac{1}{H W} \sum_{x} \sum_{y} f_{c}(x, y),
$$

$$
\begin{aligned}
& \sigma_{c}=\left\{\frac{1}{H W} \sum_{x} \sum_{y}\left\{f_{c}(x, y)-\mu_{c}\right\}^{2}\right\}^{1 / 2}, \\
& s_{c}=\left\{\frac{1}{H W} \sum_{x} \sum_{y}\left\{f_{c}(x, y)-\mu_{c}\right\}^{3}\right\}^{1 / 3},
\end{aligned}
$$

where $f_{c}(x, y)$ represents the pixel value of a component $c$ at coordinates $(x, y)$ and $H$ and $W$ are the height and width of the image region.

( 2 ) Haar Wavelet Texture

The Haar wavelet transform of the $n$-th level for onedimensional data $\left(a_{0}, a_{1}, \ldots, a_{2^{K}-1}\right)$ is formulated as

$$
\begin{aligned}
& a_{k}^{(n)}=\frac{1}{2}\left(a_{2 k}^{(n-1)}+a_{2 k+1}^{(n-1)}\right), \\
& b_{k}^{(n)}=\frac{1}{2}\left(a_{2 k}^{(n-1)}-a_{2 k+1}^{(n-1)}\right) .
\end{aligned}
$$

Here, $a_{k}^{(n)}$ and $b_{k}^{(n)}$ represent low-frequency content and high-frequency content respectively. Two-dimensional image data can be transformed by applying onedimensional functions in the horizontal direction and the vertical direction.

The Haar wavelet transform is applied in three levels to the image region. We then calculate the variance of the pixel values in each sub-band region, and these are concatenated to form the feature values.

(3) Local Binary Pattern

The local binary pattern ${ }^{20)} L_{P, R}$ from $P$ pixels on a circle of radius $R$ is formulated as

$L_{P, R}(x, y)=\left\{\begin{array}{ll}\sum_{p=0}^{P-1} \delta_{P, R}\left(x_{p}, y_{p}\right), & \text { if } U_{P, R}(x, y) \leqq 2 \\ P+1, & \text { otherwise }\end{array}\right.$.

Here, $\delta_{P, R}$ represents the magnitude relationship of intensity values between a particular pixel at $(x, y)$ and 
the surrounding pixels at $\left(x+x_{p}, y+y_{p}\right)$ and is calculated as

$\delta_{P, R}\left(x_{p}, y_{p}\right)=\left\{\begin{array}{l}1, f\left(x+x_{p}, y+y_{p}\right)-f(x, y) \geqq 0 \\ 0, \text { otherwise }\end{array}\right.$.

The values of $x_{p}$ and $y_{p}$ are given by

$$
\left\{\begin{array}{l}
x_{p}=R \cos \frac{2 \pi p}{P} \\
y_{p}=R \sin \frac{2 \pi p}{P}
\end{array} \quad(0 \leqq p \leqq P-1) .\right.
$$

The function $U_{P, R}$ in Equation (7) represents the total number of locations where there is a change between 0 and 1 in the sequence $\delta_{P, R}$ for the surrounding pixels, and is calculated by

$$
\begin{aligned}
& U_{P, R}(x, y)=\left|\delta_{P, R}\left(x_{P-1}, y_{P-1}\right)\right| \\
& \quad+\sum_{p=1}^{P-1}\left|\delta_{P, R}\left(x_{p}, y_{p}\right)-\delta_{P, R}\left(x_{p-1}, y_{p-1}\right)\right| .
\end{aligned}
$$

The proposed method calculates $L_{P, R}\left(0 \leqq L_{P, R} \leqq P+\right.$ 1) for all the pixels in the image region and obtains a frequency histogram of the results. To ensure robustness against changes in resolution, frequency histograms are calculated for each $L_{P, R}$ with $(P, R)=(8,1),(16,2)$, and $(24,3)^{20)}$.

\section{3 Extended SPM}

A conventional method ${ }^{10)}$ partitions the keyframe images horizontally and vertically into $2 \times 2$ and $1 \times 3$ grid regions, and the average feature vectors for each grid region are concatenated to obtain a feature vector for the whole image. To calculate feature vectors more robustly with respect to differences in the size and position of objects, the proposed method partitions the frame images into grid regions of various sizes. In addition, neighboring grid regions overlap each other to deal with objects that cross over the boundaries between grid regions. The average feature vectors are calculated for each grid region, and these are concatenated to form the feature vector of the entire keyframe. The specific size of the grid regions is obtained by dividing the frame image horizontally and vertically into $1 \times 1$, $2 \times 2,3 \times 3,3 \times 1,1 \times 3$, and $4 \times 4$ regions. Fig. 2 shows the grid region sizes used in the proposed method. The region sizes were determined based on the result of preliminary experiment. The amount of overlap between neighboring grid regions is $50 \%$ of the grid-region width in the horizontal direction and 50\% of the grid-region height in the vertical direction. The overlap rates were defined so that grid boundaries are covered efficiently by a small number of grids. Higher overlap rate makes it possible to generate more discriminative feature vector and to increase detection accuracy, but more computational time will be required. In contrast, lower overlap rate can reduce computational cost, but it may cause the drop of the detection accuracy.

\section{4 Training Framework based on Semi- supervised Learning}

The accuracy of a classifier is strongly dependent on the quality of the training data. However, it is very expensive to assign high-quality labels to large amounts of video data manually. The TRECVID data set ${ }^{13)}$ is labeled by collaborative annotation ${ }^{14)}$. In collaborative annotation, multiple research organizations use a Web application to label each shot. A drawback to collaborative annotation is that the criteria for determining positive examples is non-uniform due to the large number of people inputting the data. For example, with the concept "Boy", cases where people are in doubt as to whether they are looking at a positive example, on the basis of a video still frame alone, will be common.

We propose an efficient framework for generating classifier based on semi-supervised learning ${ }^{16) 17)}$, which enables to produce higher quality training data quickly and efficiently. The proposed method increases the number of positive examples while eliminating noise data by repeating the training procedure and the prediction procedure. The proposed framework is shown in Fig. 3. First, the user should prepare the initial set of positive examples. For example, the initial set can be created by performing text search on closed caption data and extracting the corresponding shots. It can be also created manually, as with the collaborative annotation of TRECVID. Then, any labels that are clearly wrong are corrected manually. Next, the classifier is trained using the corrected training data, and the classification process is applied to the original training data. The top $N$ items of the classification result are combined with $M$ items of data selected at random from the training data to form next candidate set of positive examples. The manual labeling process is repeated to correct this candidate set, the classifier is trained again, and the classification process is applied to the training data again. This is repeated until the accuracy of the classifier exceeds a certain threshold, or until the number of newly selected positive examples declines below a certain threshold. The classifier is trained in accordance with the above process. Here, random data is added when creating the candidate set of positive examples, to prevent the classifier from converging on a 


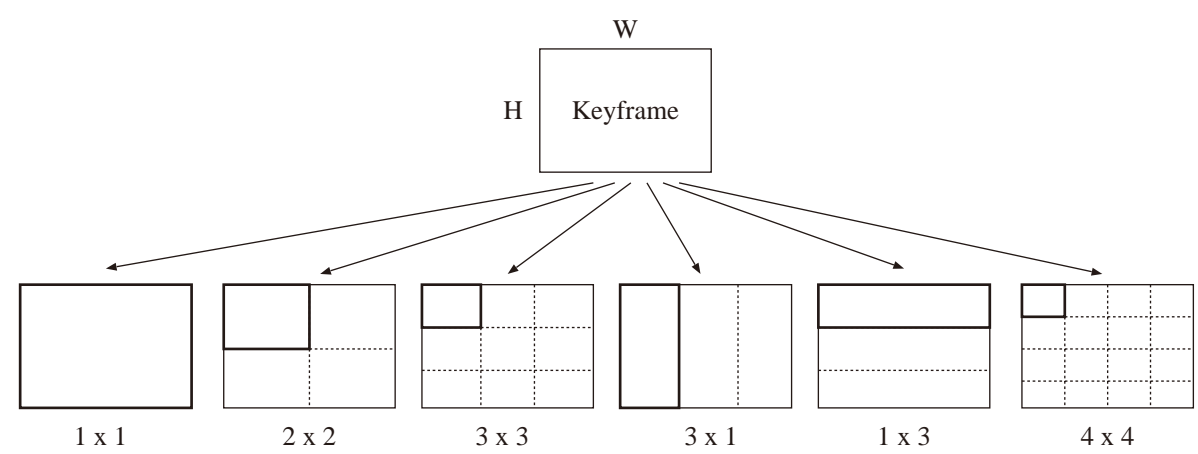

Fig. 2 Calculation of grid-based feature vector.

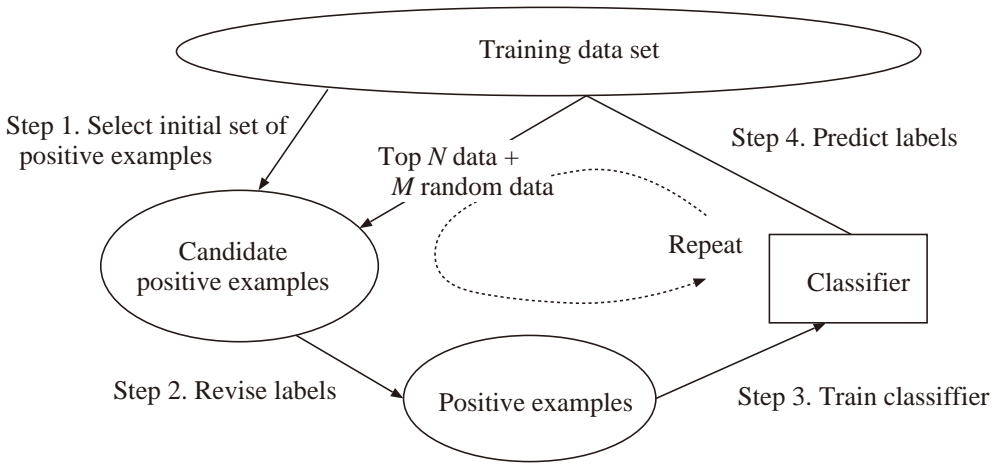

Fig. 3 Outline of training framework based on semi-supervised learning.

local solution.

\subsection{Random Forests Classifier}

Random forests ${ }^{18)}$ is a kind of ensemble learning, and it provides highly accurate classifications by using a combination of decision trees $(\mathrm{CART})^{24)}$. We use the random forests method to classify an input keyframe, because the random forests can finish both the training procedure and the prediction procedure in a short time even for high-dimension feature vectors.

However, the random forests have an issue in case of applying to imbalanced data set. The random forests algorithm works well when the training data for two classes (positive example and negative example) are roughly the same in number, but the classification error rate is rather uneven when one class is much larger than the other. The imbalance is usual with the semantic concept detection task. The conventional method resolved this problem by applying a higher weighting to the smaller class ${ }^{18)}$. However, the bootstrap samples generated by the conventional method contain few data items with a high weight and many data items with a low weight, and this situation could result in over-training. Thus, we use a modified training algorithm for creating the bootstrap samples that ensures each class is sampled with equal probability ${ }^{25)}$. The data is sampled with replacement and is not weighted.

Table 1 shows the training procedure of random forests
Table 1 Training algorithm of random forests classifier.

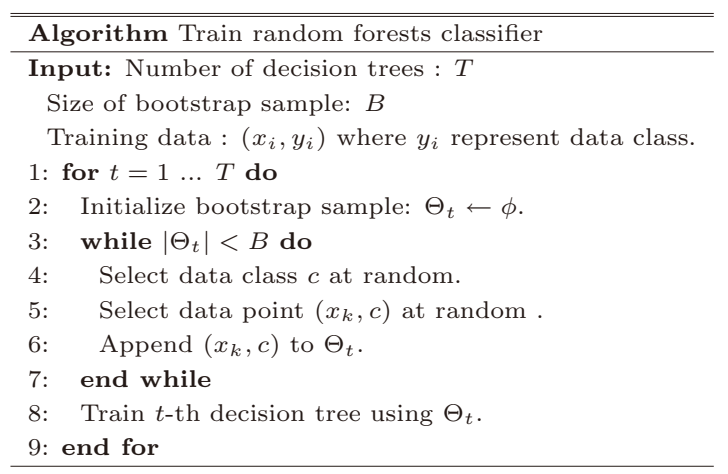

classifier. If the number of bootstrap samples is small relative to the amount of training data, the proposed method can select various data items from the minority class, making it possible to generate a classifier with high generalization capability.

\section{Experiments}

\section{1 Experiment on TRECVID data set}

(1) Settings

We performed experiments using the TRECVID 2012 13) data set to verify the effectiveness of the proposed method. The TRECVID data set is a collection of video clips published under a Creative Commons license from a variety of sources such as amateur footage and video clips from television programs ${ }^{26)}$. The data set includes approximately 27,000 video clips from ten sec- 
Table 2 Evaluation results of each method.

\begin{tabular}{c|l|l|c}
\hline \hline Method & Feature type & Training method & Mean infAP \\
\hline 1 & Ours & Semi-supervised RF & 0.106 \\
\hline 2 & Chang $^{10)}$ & Semi-supervised RF & 0.101 \\
\hline 3 & Ours & RF & 0.102 \\
\hline 4 & Chang $^{10)}$ & RF & 0.099 \\
\hline
\end{tabular}

onds to four minutes in length. Video data is encoded in MPEG-4/H.264 format. The data totals approximately 800 hours of video, of which 600 hours was used as training data, and the remaining 200 hours was used as test data. The 46 concepts defined by $\mathrm{LSCOM}^{27)}$ were used in evaluating detection accuracy. As with TRECVID, we used inferred average precision (infAP) ${ }^{28)}$ on the top 2,000 detected shots for the evaluation.

We used the four methods shown in Table 2 for comparison. The methods differ in how the feature vectors are calculated or the training method used. Methods 1 and 3 compute feature vectors using the proposed method, while methods 2 and 4 used a method due to Chang et $a .^{10)}$. Chang's method combines a BoVW method based on SIFT with conventional SPM ${ }^{8)}$. Methods 1 and 2 used the proposed semi-supervised learning with random forests ( $\mathrm{RF}$ ), while methods 3 and 4 used a conventional random forest method ${ }^{18)}$. Collaborative annotation results from TRECVID ${ }^{29)}$ were used as the initial positive candidates for the semi-supervised RF. The parameter, $N$ and $M$, described in section 2.4 were set to the value 2,000 and 200 respectively. For methods 3 and 4 , the results of collaborative annotation were used directly for training the classifier. Method 4, which uses only conventional methods, was used as the baseline method.

(2) Experimental Results

The experimental results are shown in Table 2 . The infAP value for each method is near 0.1 . The overall values are low because the average precision is calculated from thousands of detection results, regardless of the total number of positive examples included in the test data. TRECVID uses strict criterion so that more accurate comparisons can be made. For illustration, if the average precision for the top 50 shots was calculated, the accuracy result for each method would be in the range of 0.5 to 0.6 .

The evaluation results for method 4, the base line method, had the lowest accuracy, with an infAP value of 0.099. In contrast to the baseline method, method 3 , which uses the proposed feature vectors, achieved an infAP value of 0.102 . We suppose that use of the proposed features enabled appearance of the concepts to be determined more accurately. Compared to the baseline, method 2, which used the semi-supervised learning technique, achieved an infAP value of 0.101 . This is approximately the same accuracy as method 3, indicating that the improvement due to the proposed features and that due to the semi-supervised learning technique is approximately the same. We presume that through use of the semi-supervised learning technique, the quality of the training data increased, which contributed to the increased accuracy. The most accurate method was method 1, the proposed method, achieving an infAP value of 0.106 . This confirms that using a combination of the proposed feature vectors and semi-assisted learning improved detection accuracy more than either of the techniques applied separately.

Table 4 shows the results of a detailed comparison of method 1, which achieved the highest infAP, and method 4, which is the baseline method. The results show that method 1 achieved greater accuracy for 31 out of 46 concepts. The increase in accuracy was particularly large for the concepts "101 Scene_Text" and "128 Walking_Running". We conclude that the image features for these concepts were well reflected by the proposed feature values. In contrast, baseline method achieved greater accuracy for the concepts "4 Airplane_Flying" and "198 Civilian_Person". This may reflect an over-fitting problem for some concepts, due to the increased number of dimensions in the feature vectors. It may be necessary to investigate potential alternatives such as changing the feature calculation method for some concepts.

We now discuss the processing time needed to create the training data. In the collaborative annotation for TRECVID, 46 research facilities and universities applied concept labels for 346 concepts over a period of approximately 40 days. The exact amount of work time was not announced, but if we assume that each facility spent an average of two hours per day, approximately 11 hours of work was required for each concept. Our proposed method uses results of this collaborative annotation as an initial data set, and the time required for semi-supervised learning after that was approximately one hour per concept. If the initial data set can be generated more quickly, such as by analyzing closed captions, the overall amount of work required could be reduced greatly. 


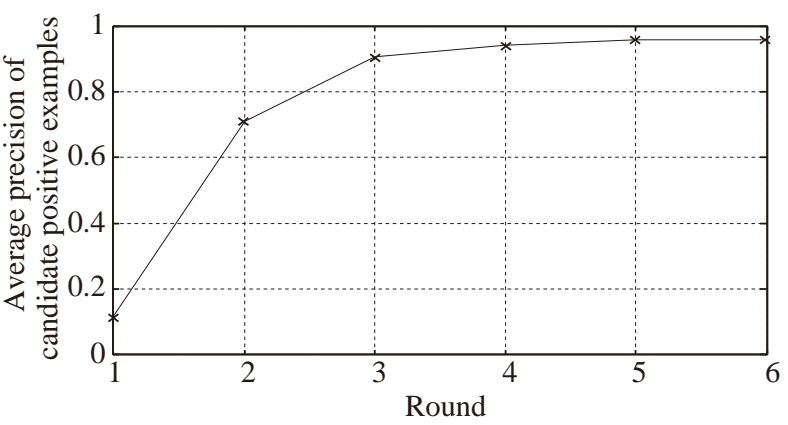

Fig. 4 Average precision of candidate positive examples while semi-supervised learning.

\section{2 Evaluation of Training Framework based on Semi-supervised Learning}

\section{(1) Settings}

We also conducted experiments to evaluate the proposed training framework using a different data set. For these experiments, we used approximately 10,000 hours of television programs that have been broadcast on NHK's General and Educational channels. These include programs in various genres and include a total of approximately two million shots. Half of these programs were used as training data, and the rest were used as test data. Of the objects defined at $\mathrm{LSCOM}^{27}$, 14 were selected to be detected. As in the previous section, the parameter, $N$ and $M$ were set to 2,000 and 200 respectively.

The initial set of positive example candidates for semi-supervised learning was created using text search on the closed captions of the programs. Specifically, the closed captions were searched for the names and related words of the concept to be detected, and a positive example label was assigned to shots where these keywords appeared as well as the shots immediately before and after. Negative example labels were assigned to any other shots. Positive example labels were assigned to the previous and following shots because in our experience, it is highly likely that the same objects will also appear in shots that are temporally very close. We also used related terms in addition to the name of the concept being detected in order to obtain a variety of candidate data.

\section{(2) Experimental Results}

Fig. 4 shows the relationship between the number of times the training process is repeated and the average precision for the trained classifier. The training data used in the training process and the data used to evaluate the average precision are the same, so it is expected that the average precision will increase as the process
Table 3 Number of positive example labels while semisupervised learning. $(N=2000, M=200)$

\begin{tabular}{c|c|c}
\hline \hline Round & $\begin{array}{c}\text { Total number of data } \\
\text { with positive example label }\end{array}$ & $\begin{array}{c}\text { Number of revised labels } \\
\text { in the round }\end{array}$ \\
\hline 1 & 111.1 & 111.1 \\
\hline 2 & 234.1 & 123.0 \\
\hline 3 & 299.9 & 65.8 \\
\hline 4 & 322.9 & 23.0 \\
\hline 5 & 328.9 & 6.0 \\
\hline 6 & 329.1 & 0.2 \\
\hline
\end{tabular}

is repeated. Our results show that it increased significantly over four repetitions, and after that the result appeared to have converged.

Table 3 shows the total number of positive example labels and the number of revised labels in each training round. Through the repeated process, the total number of positive example labels increased to about threetimes the size of the initial data set. The number of revised labels is equal to the number of positive example labels newly assigned in the round. The second round had the most frequent revision, and the number of revised labels monotonically decreased after the third round.

Approximately one hour of work was needed per concept to create the training data. Compared to the experiments in the previous section, the amount of training data increased from approximately 600 hours to approximately 5000 hours, but the time required for semisupervised learning work was about the same. This is because with the proposed method, the labels need to be corrected for only a part of the training data (step 2 of Fig. 3), so even if the amount of training data increases, the amount of work time does not. The work time will increase, however, if the value of parameter $N$, described in section 2. 4, is increased.

We now discuss detection accuracy for the proposed training framework based on semi-supervised learning. We used the average precision for the top 2,000 shots. The average precision for 14 types of concept when evaluating detection accuracy using the test data was 0.218 . In contrast, when the initial data set was used directly without performing semi-supervised learning to train classifiers, lower average precision of 0.021 was achieved. We were able to confirm that the classifier trained using the proposed method was useful, even for performing video search on a scale of 5,000 hours. We obtained particularly high accuracy, for the concepts of "Building" and "Trees", with average precision of greater than 0.5. On the other hand, the concepts "Cats" and "Dogs" achieved rates of 0.001 and 0.012 re- 
Table 4 Evaluation results for each concept.

\begin{tabular}{|c|c|c|c|}
\hline & \multirow[b]{2}{*}{ Concept } & \multicolumn{2}{|c|}{ infAP } \\
\hline & & Method 1 & $\begin{array}{l}\text { Method } 4 \\
\text { (Baseline) }\end{array}$ \\
\hline 3 & Airplane & 0.099 & 0.094 \\
\hline 4 & Airplane_Flying & 0.147 & 0.173 \\
\hline 9 & Basketball & 0.022 & 0.031 \\
\hline 13 & Bicycling & 0.011 & 0.007 \\
\hline 15 & Boat_Ship & 0.051 & 0.050 \\
\hline 16 & Boy & 0.027 & 0.024 \\
\hline 17 & Bridges & 0.018 & 0.026 \\
\hline 25 & Chair & 0.033 & 0.039 \\
\hline 31 & Computers & 0.009 & 0.004 \\
\hline 51 & Female_Person & 0.194 & 0.187 \\
\hline 54 & Girl & 0.021 & 0.010 \\
\hline 56 & Government-Leader & 0.162 & 0.123 \\
\hline 57 & Greeting & 0.051 & 0.056 \\
\hline 63 & Highway & 0.030 & 0.035 \\
\hline 71 & Instrumental_Musician & 0.093 & 0.080 \\
\hline 72 & Kitchen & 0.010 & 0.009 \\
\hline 74 & Landscape & 0.334 & 0.328 \\
\hline 75 & Male_Person & 0.616 & 0.620 \\
\hline 77 & Meeting & 0.084 & 0.063 \\
\hline 80 & Motorcycle & 0.057 & 0.044 \\
\hline 84 & Nighttime & 0.042 & 0.049 \\
\hline 85 & Office & 0.076 & 0.080 \\
\hline 95 & Press_Conference & 0.030 & 0.025 \\
\hline 99 & Roadway_Junction & 0.034 & 0.022 \\
\hline 101 & Scene_Text & 0.073 & 0.011 \\
\hline 105 & Singing & 0.018 & 0.029 \\
\hline 107 & Sitting_Down & 0.002 & 0.002 \\
\hline 112 & Stadium & 0.076 & 0.090 \\
\hline 116 & Teenagers & 0.041 & 0.030 \\
\hline 120 & Throwing & 0.018 & 0.018 \\
\hline 128 & Walking_Running & 0.222 & 0.163 \\
\hline 155 & Apartments & 0.059 & 0.056 \\
\hline 163 & Baby & 0.038 & 0.033 \\
\hline 198 & Civilian_Person & 0.733 & 0.756 \\
\hline 199 & Clearing & 0.089 & 0.097 \\
\hline 254 & Fields & 0.175 & 0.161 \\
\hline 267 & Forest & 0.117 & 0.090 \\
\hline 274 & George_Bush & 0.087 & 0.054 \\
\hline 276 & Glasses & 0.051 & 0.050 \\
\hline 297 & Hill & 0.105 & 0.090 \\
\hline 321 & Lakes & 0.124 & 0.137 \\
\hline 338 & Man_Wearing_A_Suit & 0.169 & 0.138 \\
\hline 342 & Military_Airplane & 0.085 & 0.082 \\
\hline 359 & Oceans & 0.170 & 0.173 \\
\hline 434 & Skier & 0.108 & 0.074 \\
\hline 440 & Soldiers & 0.046 & 0.045 \\
\hline & Average & 0.106 & 0.099 \\
\hline
\end{tabular}

spectively. For recognition of highly specific concepts, it seems that not only the training method, but the type of features used for classification must be considered.

The experimental result shows that the proposed framework can reduce the large amount of work time to create classifier without drastic decrease of the detection accuracy comparing conventional training method.

\section{Conclusion}

In this paper, we proposed a method for calculating feature vectors that is robust against variation in the size or position of objects by combining image features calculated for block regions of various sizes. We also proposed a method for using semi-supervised learning to remove error from the training data. Evaluation results showed that the proposed method provides better detection accuracy than earlier methods. In future work, we intend to continue improving the image feature vector in order to further improve accuracy and to incorporate audio features in addition to the video features.

\section{References}

1) J. Sivic and A. Zisserman: "Video Google: A Text Retrieval Approach to Object Matching in Videos", Proc. ICCV'03, 2, pp. 1470-1477 (2003)

2) G. Csurka, C. Bray, C. Dance and L. Fan, "Visual Categorization with Bags of Keypoints", Proc. ECCV Workshop on SLCV, pp. 59-74 (2004)

3) D.G. Lowe, "Object Recognition from Local Scale-invariant Features", Proc. ICCV'99, 2. pp. 1150-1157 (1999)

4) H. Bay, A. Ess, T. Tuytelaars and L.V. Gool, "SURF: Speeded Up Robust Features", Computer Vision and Image Understanding, 110, 3, pp. 346-359 (2008)

5) "TRECVID Workshop Notebook Papers/Slides", http://wwwnlpir.nist.gov/projects/tvpubs/tv.pubs.org.html

6) J. Wang, J. Yang, K. Yu, F. Lv, T. Huang and Y. Gong, "Locality-constrained Liner Coding for Image Classification", Proc. IEEE CVPR'10, pp. 3360-3367 (2010)

7) L. Xie, Q. Tian and B. Zhang, "Spatial Pooling of Heterogeneous Features for Image Applications", Proc. ACM Multimedia, pp. 539-548 (2012)

8) S. Lazebnik, C. Schmid, and J. Ponce, "Beyond Bags of Features: Spatial Pyramid Matching for Recognizing Natural Scene Categories", Proc. CVPR'06, pp. 2169-2178 (2006)

9) J. Yang, K. Yu, Y. Gong, and T. Huang, "Linear Spatial Pyramid Matching using Sparse Coding for Image Classification", Proc. IEEE CVPR'09, pp. 1794-1801 (2009)

10) S.-F. Chang, J. He, Y.-G. Jiang, E.E. Khoury, C.-W. Ngo, A. Yanagawa and E. Zavesky, "Columbia University/VIREOCity/IRIT TRECVID2008 High-level Feature Extraction and Interactive Video Search", Proc. TRECVID Workshop (2008)

11) L. Fei-Fei, R. Fergus, and P. Perona, "Learning Generative Visual Models from Few Training Examples: An Incremental Bayesian Approach Tested on 101 Object Categories", Proc. Computer Vision and Image Understanding, 1, 106, pp. 59-70 (2007)

12) G. Griffin, A. Holub, and P. Perona, "Caltech-256 Object Category Dataset" (2007)

13) "TREC Video Retrieval Evaluation: TRECVID", http:// trecvid.nist.gov/

14) S. Ayache and G. Quenot, "Video Corpus Annotation using Active Learning", Proc. ECIR'08, pp. 187-198 (2008)

15) X. Zhu, "Semi-supervised Learning Literature Survey", Computer Science TR-1530, University of Wisconsin Madison (2008)

16) D. Yarowsky, "Unsupervised Word Sense Disambiguation Rivaling Supervised Methods", Proc. ACL'95, pp. 189-196 (1995)

17) B. Maeireizo, D. Litman and R. Hwa, "Co-training for Predicting Emotions with Spoken Dialogue Data", Proc. ACL'04, pp. 203-206 (2004)

18) L. Breiman, "Random Forests", Machine Learning, 45, pp. 5-32 (2001)

19) Y. Kawai, H. Sumiyoshi, and N. Yagi, "Shot Boundary Detection at TRECVID 2007", Proc. TRECVID Workshop, pp. 277-284 (2007)

20) T. Ojala M. Pietikaninen and T. Maenpaa, "Multiresolution Gray-scale and Rotation Invariant Texture Classification with Local Binary Patterns", IEEE Trans. Pattern Analysis and Machine Intelligence, 24, 7, pp. 971-987 (2002)

21) F. Perronnin, J. Sanchez and T. Mensink, "Improving the Fisher Kernel for Large-scale Image Classification", Proc. ECCV'10, vol. 6314, pp. 143-156 (2010)

22) J.R.R. Uijlings, A.W.M. Smeulders and R.J.H. Scha, "Real-time Visual Concept Classification", IEEE Trans. Multimedia, 12, 7 , pp. 665-681 (2010) 
23) Y.-G. Jiang, C.-W. Hgo and J. Yang, "Towards Optimal Bagof-features for Object Categorization and Semantic Video Retrieval", Proc. ACM CIVR'07, pp. 494-501 (2007)

24) L. Breiman, J.H. Friedman, R.A. Olshen and C.J. Stone, "Classification and Regression Trees", Wadsworth and Brooks (1984)

25) Y. Kawai, M. Takahashi, M. Fujii, M. Naemura and S. Sato, "NHK STRL at TRECVID 2010: Semantic Indexing and Surveillance Event Detection", Proc. TRECVID Workshop, 1, pp. 304310 (2010)

26) Internet Archive, http://archive.org/

27) M. Naphade, J.R. Smith, J. Tesic, S-F. Chang, W. Hsu, L. Kennedy, A. Hauptmann, and J. Curtis, "Large-scale Concept Ontology for Multimedia", IEEE Trans. Multimedia, 13, 3, pp. 86-91 (2006)

28) E. Yilmaz, E. Kanoulas, and J.A. Aslam, "A Simple and Efficient Sampling Method for Estimating AP and NDCG", Proc. ACM SIGIR conference on Research and development in information retrieval, pp. 603-610 (2008)

29) "Availability of Data Sets used in TRECVID", http:// trecvid.nist.gov/trecvid.data.html

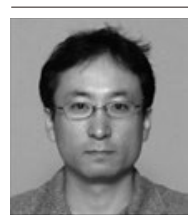

Yoshihiko Kawai received the B.E. and M.E. degree in engineering science, and the Ph.D. degree in engineering from Osaka University, Osaka, Japan, in 1999, 2001, and 2010, respectively. He is currently a principal research engineer in NHK Science and Technology Research Laboratories. His research interests include multimedia processing and video indexing. He is a member of IEICE and ITE.

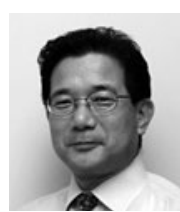

Mahito Fujii received the B.E. degree and the M.E. degree in electrical engineering from Nagoya University, Nagoya, Japan, in 1981 and 1983 respectively. Originally joining NHK in 1983, he has worked with their Science and Technical Research Laboratories since 1987, engaging in neural networks and pattern recognition fields. From 1993 to 1994 , he was a visiting researcher at the Robotics Institute of Carnegie Mellon University, Pittsburgh PA, USA. From 1998 to 2001, he headed the department 5 at ATR (Advanced Telecommunication Research Institute International) Human Information Processing Research Laboratories, Kyoto, Japan. He received the Ph.D. degree in information science from Nagoya University, Nagoya, Japan, in 2008. $\mathrm{He}$ is a senior research engineer at NHK Science and Technology Research Laboratories. He is a member of IEEE, IEICE and ITE. 